\title{
Lossless Snubber with Minimum Voltage Stress for Continuous Current Mode Tapped-Inductor Boost Converters for High Step-up Applications
}

\author{
Jeong-il Kang ${ }^{\dagger}$, Sang-Kyoo Han ${ }^{*}$, and Jonghee Han ${ }^{* *}$ \\ ${ }^{\dagger * *}$ Research \& Development Office, Visual Display, Samsung Electronics Co., Ltd., Suwon, Korea \\ "School of Electrical Engineering, College of EECS, Kookmin University, Seoul, Korea
}

\begin{abstract}
To invigorate the tapped-inductor boost (TIB) topology in emerging high step-up applications for off-grid products, a lossless snubber consisting of two capacitors and three diodes is proposed. Since the switch voltage stress is minimized in the proposed circuit, it is allowed to use a device with a lower cost, higher efficiency, and higher availability. Moreover, since the leakage inductance is fully utilized, no effort to minimize it is required. This allows for a highly productive and cost-effective design of the tapped-inductor. The proposed circuit also shows a high step-up ratio and provides relaxation of the switching loss and diode reverse-recovery. In this paper, the operation is analyzed in detail, the steady-state equation is derived, and the design considerations are discussed. Some experimental results are provided to confirm the validity of the proposed circuit.
\end{abstract}

Key words: Current control, DC-DC power conversion, Efficiency, Snubber circuit

\section{INTRODUCTION}

In Sub-Saharan African and South Asian underdeveloped countries, one can easily find the off-grid areas where people do not have an access to the power grid. In those areas, the demand for stand-alone off-grid power systems based on renewable energy such as sunlight and wind is rapidly increasing. This reflects the recent increase in their need for electricity. Market analysts have found that there are already more than 2 million off-grid power systems. The output voltage of these kinds of power systems is generally very low when compared to that coming from the ac mains of the power grid. They are mostly loaded by $12 \mathrm{~V}$ automotive batteries for a continuous supply of electricity under varying weather conditions [1]-[4].

Market analysts have also found that the main use of this off-grid power is for watching television and lighting as shown in Fig. 1. The lighting devices should be made of highly

Manuscript received Dec. 10, 2013; accepted Apr. 2, 2014

Recommended for publication by Associate Editor Bor-Ren Lin.

${ }^{\dagger}$ Corresponding Author: jeongil.kang@samsung.com

Tel: +82-31-277-2984, Samsung Electronics Co., Ltd.

*School of Electrical Engineering, College of EECS, Kookmin

University, Korea

${ }^{* *}$ Research \& Development Office, Visual Display, Samsung Electronics Co., Ltd., Korea efficient LEDs since this electric power is seriously limited by the weather conditions and battery capacity. For the same reason, the televisions should be LCD televisions with an LED backlight unit since they use less power than any other type of television. These devices usually consist of 30 or more LEDs. For example, a $30 \mathrm{~W}$-rated medium power LED tube and a backlight unit smaller than 32-inchs contain around 30 LEDs since $1 \mathrm{~W}$-LEDs are currently in wide use. It needs to be noted that all of the LEDs are preferred to be connected in series and driven by a single circuit so that all of the LEDs carry the same current for good brightness uniformity. A challenge for the circuit engineer is that this configuration requires a 10-times or higher step-up LED driver, which is practically unattainable with conventional boost topologies due to the duty ratio being very close to the unity [5].

One simple and effective alternative to overcome the step-up ratio limitation in boost topologies is the tapped-inductor boost (TIB) topology. By simply replacing the inductor with a tapped-inductor, the TIB topology gives a much higher step-up ratio at a reduced duty ratio [5]. Another important advantage of the TIB topology is that the voltage stress on the switch in its turn-off state is equal to the mean of the input and output voltages weighted by the winding turn numbers, whereas a voltage equal to the output voltage appears on the switch in the 


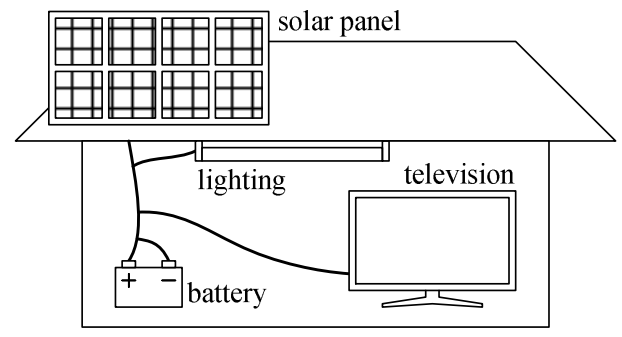

Fig. 1. Typical off-grid power appliances.

conventional boost topology. However, this feature is limited to cases where the effects due to parasitic reactance are negligible. In practice, the switch of the TIB topology suffers from a severe voltage spike due to the leakage inductance of the tapped-inductor. Without suppressing this voltage spike, the TIB topology is much the worse from the standpoint of the switching stress. There have been many elaborated studies on limiting the switch voltage spike of the TIB topology and improving the converter performance [6]-[18]. However, they generally modify the original TIB topology, limit the switch voltage to a non-minimum value, or require an auxiliary inductor or winding to achieve proper circuit operation.

In this paper, a lossless snubber with minimum voltage stress for a continuous current mode TIB converter is proposed [19]. It consists of two capacitors and three diodes. This provides more boost capability and a higher efficiency to the original TIB converter. The following features make the proposed circuit very attractive especially in commercial applications. First, a large improvement in the circuit reliability is achieved. Since the proposed circuit limits the switch voltage of the TIB converter to its minimum, it is possible to use switches with significantly lower voltage ratings which generally results in a lower cost, higher performance, and higher availability. Second, by utilizing the leakage inductance of the tapped-inductor, problems related to $d i / d t$ such as the switching loss and the diode reverse-recovery are alleviated. Moreover, efforts in designing magnetically coupled devices to minimize the flux leakage, such as special winding methods which in most cases sacrifice cost and productivity, are not necessary. Third, the proposed circuit can be readily incorporated into an existing system since it does not modify the original TIB topology.

The operation principle of the proposed circuit is analyzed in detail in this paper, and the steady-state step-up relationship is derived based on this analysis. The design considerations are also discussed with various boundary conditions related to the proper operation. Some experimental results from a $12 \mathrm{~V}$ to $120 \mathrm{~V} / 240 \mathrm{~mA}$ LED driver prototype for a lighting module are also provided to confirm the validity of the proposed circuit.

\section{CIRCUIT ANALYSIS}

\section{A. Operation analysis}

The proposed circuit consists of a clamp capacitor $C_{c}$, a

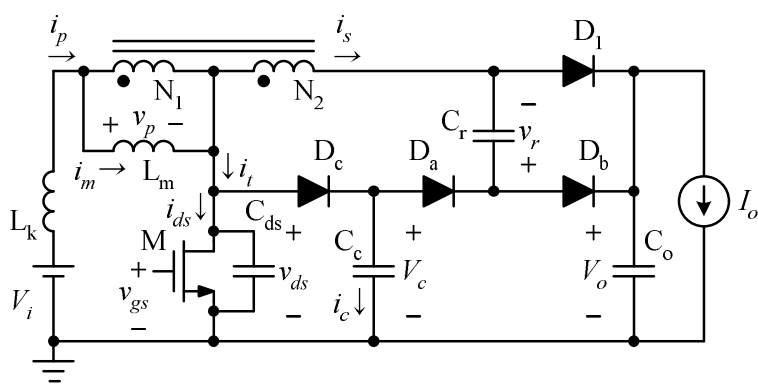

Fig. 2. TIB converter with proposed lossless snubber circuit.

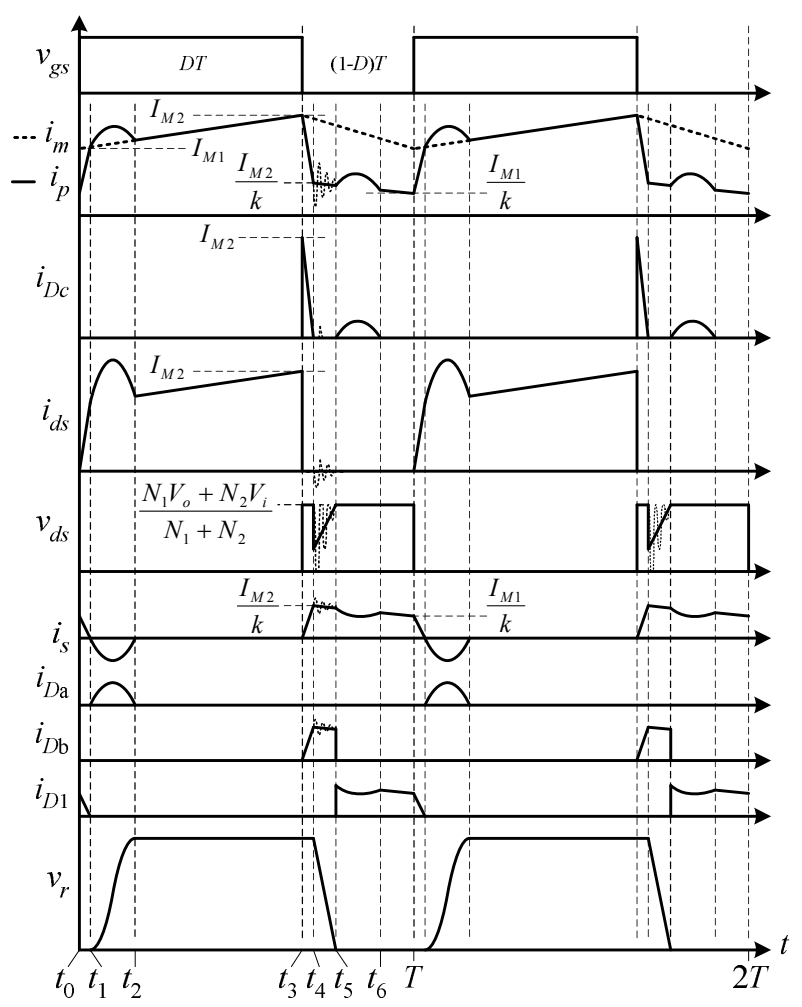

Fig. 3. Qualitative illustration of theoretical key waveforms.

clamp diode $D_{c}$, a resonant capacitor $C_{r}$, and two path diodes $D_{a}$ and $D_{b}$ for charging and discharging $C_{r}$, as shown in Fig. 2. The tapped-inductor is modeled as an ideal transformer with a turns ratio of $N=N_{2} / N_{1}$, a magnetizing inductance $L_{m}$, and a leakage inductance $L_{k}$. The switch $M$ is modeled as an ideal switch in parallel with the output capacitance $C_{d s}$. The parasitic elements other than $L_{m}, L_{k}$ and $C_{d s}$ are basically ignored. However, they are separately considered when they produce a meaningful effect. The switching frequency is assumed to be $f=1 / T$, and $1+N$ is denoted as $k$ for convenience in deriving the equations.

Fig. 3 shows the key waveforms of the proposed converter (referring to the TIB converter with the proposed snubber). The magnetizing current $i_{m}$ is shown to keep varying throughout the switching cycle. However, the operation is analyzed with the assumption that it is constant during the switching transients 


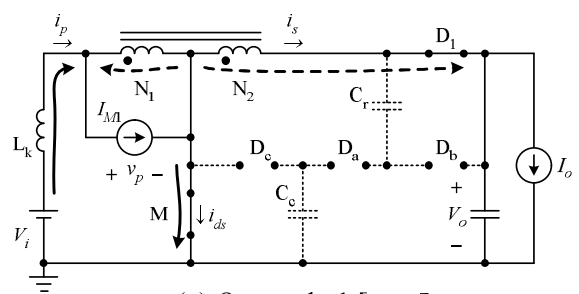

(a) On-mode $1\left[t_{0} \sim t_{1}\right]$

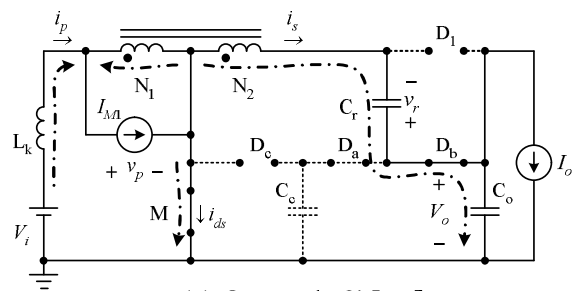

(c) On-mode 2' $\left[t_{2}+\right]$

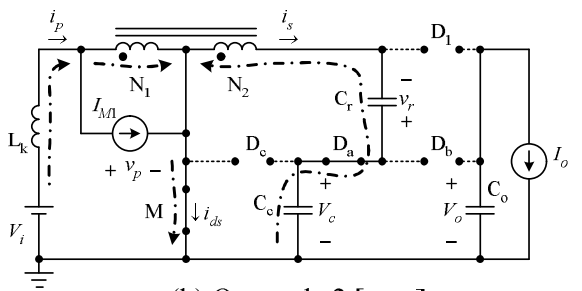

(b) On-mode $2\left[t_{1} \sim t_{2}\right]$

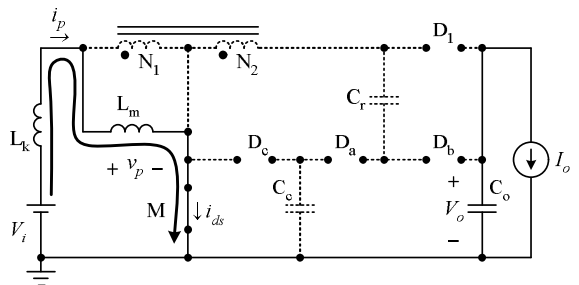

(d) On-mode $3\left[t_{2} \sim t_{3}\right]$
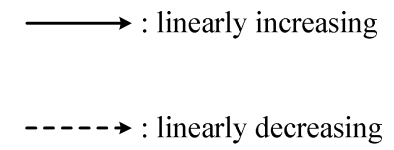

Fig. 4. Topological stages in On-modes.

and snubber operation. In other words, $i_{m}=I_{M 1}$ for $t_{0} \sim t_{2}$ and $i_{m}=I_{M 2}$ for $t_{3} \sim t_{6}$, where $I_{M 1}$ and $I_{M 2}$ denote the minimum and maximum values of $i_{m}$, respectively. Otherwise, the quantitative analysis becomes very complicated. The input voltage $V_{i}$, output voltage $V_{o}$, output current $I_{o}$, and clamp voltage $V_{c}$ are assumed to be ripple-free and constant. It is also assumed that $C_{r}$ is much larger than $C_{d s}$. The clamp voltage $V_{c}$ which determines the switch voltage stress can be obtained from the input and output voltages weighted by the tapped-inductor turns ratios as:

$$
V_{c}=\frac{N_{1} V_{o}+N_{2} V_{i}}{N_{1}+N_{2}} .
$$

There are 7 topological states in one switching cycle of the proposed converter.

Pre-On-mode $\mathbf{1}\left[\sim \boldsymbol{t}_{\mathbf{0}}, \boldsymbol{t}_{\mathbf{6}} \sim \boldsymbol{T}\right]$ : The switch $M$ is in the off-state, and the resonant capacitor $C_{r}$ is in its completely discharged state. The secondary current of $i_{m} / k$, which is equal to the primary current, is carried by output diode $D_{1}$, and the magnetizing current $i_{m}$ decreases with a slope of $\left(V_{i}-V_{c}\right) / L_{m}$. This mode is identical to the switch-off mode of the original TIB converter.

On-mode $1\left[\boldsymbol{t}_{0} \sim \boldsymbol{t}_{1}\right]$ : This mode begins with the turn-on of the switch $M$ and represents a turn-on delay due to the current slopes which are limited by the leakage inductance $L_{k}$. The equivalent circuit is shown in Fig. 4(a).

Since the switch current $i_{d s}$ is slanted with a slope limited by $L_{k}$, the zero current soft turn-on of $M$ is achieved, alleviating the switch turn-on loss. This mode ends when the secondary current $i_{s}$ reaches zero, providing zero current soft turn-off of output diode $D_{1}$.

The reverse-recovery current, which is not obvious in Fig. 3, is illustrated in detail in Fig. 5. Since the slope of $i_{s}$ is also limited by $L_{k}$, the peak reverse-recovery current of $D_{1}$, denoted by $-I_{r r 1}$, is small, providing relaxation of the associated noise and EMI problems. This reverse-recovery current slightly further discharges $L_{k}$ and leaves a small negative current $-I_{r r 1}$ in the secondary winding at the end of this mode.

* Key equations for On-mode 1:

$$
\begin{gathered}
i_{p}(t)=\frac{I_{M 1}}{k}+\frac{1}{L_{k}}\left(V_{i}+\frac{V_{o}}{N}\right) t, \\
i_{s}(t)=\frac{I_{M 1}}{k}-\frac{1}{L_{k} N}\left(V_{i}+\frac{V_{o}}{N}\right) t, \\
i_{d s}(t)=\frac{k}{L_{k} N}\left(V_{i}+\frac{V_{o}}{N}\right) t,
\end{gathered}
$$

where $i_{m}(t)=I_{M 1}$.

On-mode $2\left[\boldsymbol{t}_{1} \sim \boldsymbol{t}_{2}\right]$ : The small negative current of $-I_{r r 1}$ in the secondary winding, which is generated by the reverse-recovery of $D_{1}$ at the end of On-mode 1, changes its path from $D_{1}$ to $D_{a}$ at $t=t_{1}$, and a path for the resonance of $C_{r}$ and $L_{k}$ to charge $C_{r}$ is formed, as shown in Fig. 4(b). Neglecting the small jump of $I_{r r 1}$ in the waveform of $i_{D a}$ at $t=t_{1}$, shown in Fig. 5, the resonance of $C_{r}$ and $L_{k}$ provides the zero current soft turn-on of $D_{a}$ by slowing down the current rise.

* Key equations for On-mode 2:

$$
\begin{gathered}
i_{p}(t)=I_{M 1}+N \sqrt{\frac{C_{r}}{L_{k}}}\left(V_{i}+\frac{V_{c}}{N}\right) \sin \omega_{1}\left(t-t_{1}\right), \\
i_{s}(t)=-\sqrt{\frac{C_{r}}{L_{k}}}\left(V_{i}+\frac{V_{c}}{N}\right) \sin \omega_{1}\left(t-t_{1}\right), \\
i_{d s}(t)=I_{M 1}+k \sqrt{\frac{C_{r}}{L_{k}}}\left(V_{i}+\frac{V_{c}}{N}\right) \sin \omega_{1}\left(t-t_{1}\right), \\
v_{r}(t)=\left(N V_{i}+V_{c}\right)\left\{1-\cos \omega_{1}\left(t-t_{1}\right)\right\},
\end{gathered}
$$


where $\omega_{1}=1 /\left\{N \cdot\left(L_{k} C_{r}\right)^{1 / 2}\right\}$, and $i_{m}(t)=I_{M 1}$.

After a half cycle of resonance, the voltage across $C_{r}$ reaches its peak value obtained from (8) as:

$$
v_{r}\left(t_{2}\right)=2\left(N V_{i}+V_{c}\right)
$$

and the secondary current reaches zero providing the zero current soft turn-off of $D_{a}$ at $t=t_{2}$. This is the end of On-mode 2.

The amount of charge released from $C_{c}$ to charge $C_{r}$ during this mode can be obtained from (6) as:

$$
Q_{o}=\int_{t_{1}}^{t_{2}}-i_{s}(t) d t=2 C_{r}\left(N V_{i}+V_{c}\right)
$$

As illustrated in Fig. 5, the reverse-recovery of $D_{a}$ slightly further discharges $L_{k}$ and leaves a small positive current $I_{r r 2}$ in the secondary winding. This current turns on $D_{b}$, resulting in the equivalent circuit shown in Fig. 4(c). The slope of $i_{s}$ at this moment can be obtained from (6) as:

$$
\frac{d i_{s}}{d t}\left(t_{2}\right)=\frac{1}{L_{k} k}\left\{V_{i}\left(\frac{3}{N}+1\right)-\frac{V_{o}}{N}\left(1-\frac{1}{N}\right)\right\} .
$$

If the sign of (11) is negative, the current $i_{s}$ cannot increase any further beyond $I_{r r 2}$ but immediately starts decreasing toward zero at $t>t_{2}$, as shown in Fig. 5. After this current comes back to zero, the path diode $D_{b}$ processes its reverse-recovery and then turns back off, leaving a small negative current in the secondary winding. This small negative current brings back the equivalent circuit shown in Fig. 4(b) by turning on $D_{a}$ again. Then the current $i_{s}$ immediately restarts increasing toward zero, repeating the phase just before the first turn-off of $D_{a}$. In this case, the path diodes $D_{a}$ and $D_{b}$ repeatedly turn on and off, and the secondary current $i_{s}$ settles to zero, eventually making both of the diodes remain in the turn-off state.

If the sign of (11) is positive, the current $i_{s}$ further increases beyond $I_{r r 2}$ after the turn-on of $D_{b}$. In this case, the proposed converter maintains the equivalent circuit shown in Fig. 4(c), and $L_{k}$ and $C_{r}$ start another half-cycle of resonance to discharge $C_{r}$ from the peak voltage given by (9). Since there is no benefit to this extra resonance, this paper suggests avoiding it by making (11) negative in pursuit of simple and efficient operation.

On-mode $3\left[\boldsymbol{t}_{\mathbf{2}} \sim \boldsymbol{t}_{3}\right]$ : This mode is equivalent to the switch-on mode of the original TIB converter, as shown in Fig. 4(d). All of the diodes are in the off-state, and the magnetizing current $i_{m}$ increases with a slope of $V_{i} / L_{m}$ and finally reaches $I_{M 2}$ at the end of this mode at $t=t_{3}$.

Off-mode $1\left[\boldsymbol{t}_{3} \sim \boldsymbol{t}_{4}\right]$ : This mode begins with the turn-off of $M$

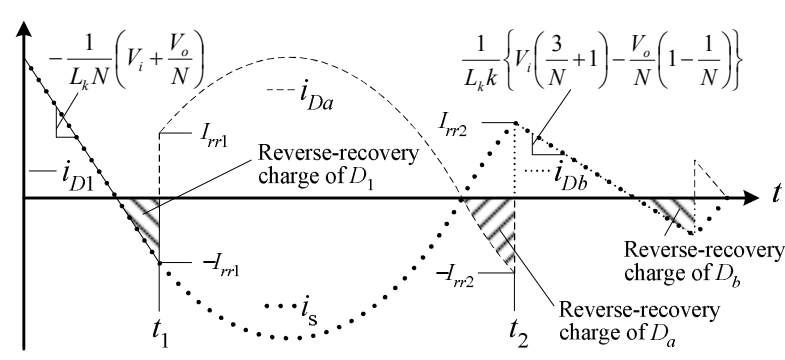

Fig. 5. Reverse-recovery currents of diodes.

at $t=t_{3}$. Neglecting the output capacitance of the diode, the path diode $D_{b}$ turns on immediately, and the leakage inductance $L_{k}$ starts resonating with $C_{d s}$ to charge $C_{d s}$ up to the clamp voltage $V_{c}$ with its initial current of $I_{M 2}$. However, this resonance can also be neglected since its characteristic impedance $\left(L_{k} / C_{d s}\right)^{1 / 2}$, where the value of $L_{k}$ is in most cases much larger than that of $C_{d s}$, can be assumed to be so much larger than 1 that there is negligible change in the resonant current from the initial value of $I_{M 2}$ while the voltage across $C_{d s}$ makes a large swing from zero to $V_{c}$. Therefore, this mode can be assumed to begin with the equivalent circuit shown in Fig. 6(a), where the capacitance $C_{d s}$ is also neglected since it is in parallel with a much larger-valued $C_{c}$.

During this mode, the energy stored in $L_{k}$ is discharged into $C_{c}$, and the primary current is transferred to the secondary side with a slope limited by $L_{k}$. To simplify the analysis, from this point on the voltage $v_{r}$ is assumed to maintain a constant value given by (9) during this mode.

* Key equations for Off-mode 1:

$$
i_{D c}(t)=I_{M 2}-\frac{2}{L_{k}}\left(V_{i}+\frac{V_{c}}{N}\right)\left(1+\frac{1}{N}\right)\left(t-t_{3}\right),
$$

where $i_{m}(t)=I_{M 2}$, and $i_{D c}(t)$ is the current flowing through $D_{c}$.

This mode ends when all of the energy in $L_{k}$ is discharged into $C_{c}$, and (12) becomes zero providing the zero current soft turn-off of $D_{c}$.

Off-mode $2\left[t_{4} \sim t_{5}\right]$ : This mode consists of two components: one is the high-frequency ac component caused by the resonance of $C_{d s}$ and $L_{k}$ beginning with a fast decrease in $v_{d s}$ from $V_{c}$, and the other one is the linear dc component representing a slow increase in $v_{d s}$ caused by the discharge of $C_{r}$ to the output. These two components are superposed so that the ac component is centered at the dc component, as shown in the interval of $t_{4} \sim t_{5}$ in Fig. 3, where the ac components are shown by dotted lines.

The peak of the $v_{d s}$ waveform is bounded to $V_{c}$ by $D_{c}$ and $C_{c}$, while the valley is bounded to the ground by the body diode of $M$. As the dc component in $v_{d s}$ increases, the ac component is pulled up toward the clamp voltage $V_{c}$, which results in the repetitive clamping of the ac component of $v_{d s}$ to $V_{c}$. Therefore, 


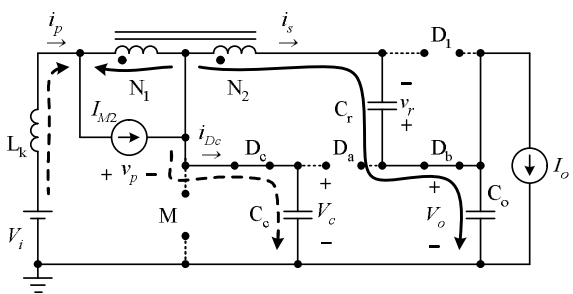

(a) Off-mode $1\left[t_{3} \sim t_{4}\right]$

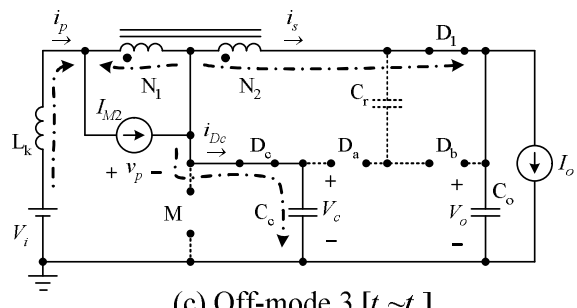

(c) Off-mode $3\left[t_{5} \sim t_{6}\right]$

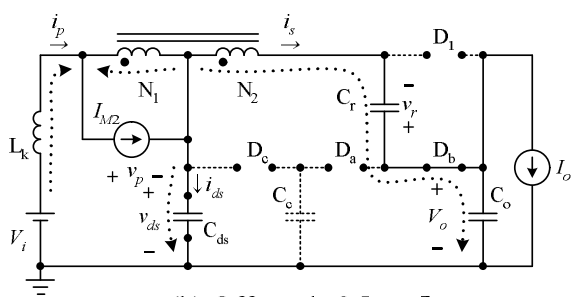

(b) Off-mode $2\left[t_{4} \sim t_{5}\right]$

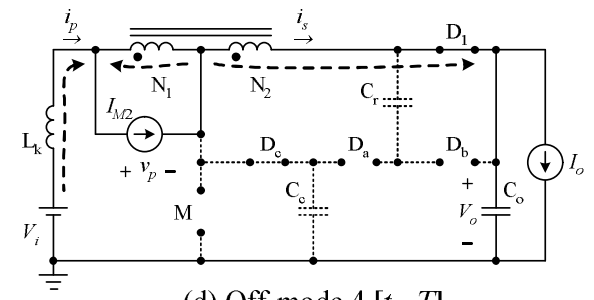

(d) Off-mode $4\left[t_{6} \sim T\right]$

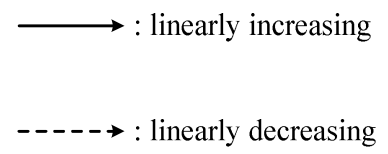

-.-.-. : resonant

Fig. 6. Topological stages in Off-modes.

the ac component as well as the associated parasitic losses in the circuit decay rapidly.

By neglecting the ac component, the equivalent circuit of this mode becomes as shown in Fig. 6(b). This mode ends when $C_{r}$ is completely discharged, and the voltages across the switch $M$ and output diode $D_{1}$ reach $V_{c}$ and zero, respectively.

* Key equations for Off-mode 2:

$$
\begin{gathered}
i_{p}(t)=i_{s}(t)=\frac{I_{M 2}}{k}, \\
v_{r}(t)=2\left(N V_{i}+V_{c}\right)-\frac{I_{M 2}}{k C_{r}}\left(t-t_{4}\right), \\
v_{d s}(t)=\frac{N V_{c}-V_{c}-2 N V_{i}}{1+N}+\frac{I_{M 2}}{k^{2} C_{r}}\left(t-t_{4}\right),
\end{gathered}
$$

where $i_{m}(t)=I_{M 2}$.

The duration of this mode, denoted by $t_{45}$, can be obtained from (14) as:

$$
t_{45}=\frac{2 k C_{r}}{I_{M 2}}\left(N V_{i}+V_{c}\right)
$$

Off-mode $3\left[\boldsymbol{t}_{\mathbf{5}} \sim \boldsymbol{t}_{\mathbf{6}}\right]$ : The secondary current that was flowing through $D_{b}$ is switched to $D_{1}$ at $t=t_{5}$, providing the zero voltage soft turn-on of $D_{1}$. The equivalent circuit is shown in Fig. 6(c), where $C_{d s}$ in parallel with $C_{c}$ is neglected. In this mode, the clamp capacitor $C_{c}$ resonates with $L_{k}$, and the charge lost by $C_{c}$ in On-mode 2 is replenished. This mode ends when the diode $D_{c}$ turns off with zero current after a half-cycle of resonance, resulting in the soft reset of the proposed snubber.

Since the assumption of a constant voltage on $C_{c}$ makes it inappropriate to characterize this mode by setting up and solving differential equations, the charge balance in $C_{c}$ is considered instead. Assume a sine current whose amplitude is unknown but whose angular frequency is equal to $k /\left\{N \cdot\left(L_{k} C_{c}\right)^{1 / 2}\right\}$, a half-cycle integration of this current flowing into $C_{c}$ can be balanced with (10) to give the following key equations.

* Key equations for Off-mode 3:

$$
\begin{gathered}
i_{D c}(t)=\frac{k C_{r}}{\sqrt{L_{k} C_{c}}}\left(V_{i}+\frac{V_{c}}{N}\right) \sin \omega_{2}\left(t-t_{5}\right), \\
i_{p}(t)=\frac{I_{M 2}}{k}+\frac{C_{r}}{\sqrt{L_{k} C_{c}}}\left(N V_{i}+V_{c}\right) \sin \omega_{2}\left(t-t_{5}\right), \\
i_{s}(t)=\frac{I_{M 2}}{k}-\frac{C_{r}}{\sqrt{L_{k} C_{c}}}\left(V_{i}+\frac{V_{c}}{N}\right) \sin \omega_{2}\left(t-t_{5}\right),
\end{gathered}
$$

where $\omega_{2}=k /\left\{N \cdot\left(L_{k} C_{c}\right)^{1 / 2}\right\}$, and $i_{m}(t)=I_{M 2}$.

It should be noted that the charge gained by $C_{c}$ through the clamping of $v_{d s}$ in Off-modes 1 and 2, which is not considered in (17)-(19), in practice, has the effect of reducing the amount of charge that should be replenished in this mode for the snubber reset, consequently reducing the amplitudes of the sine terms in (17)-(19). The larger the charge gained in Off-modes 1 and 2, the smaller the peak of the resonant currents in Off-mode 3. This makes the operation of the circuit in this mode less affected by the snubber. Therefore, it can be said that the neglect of the charge gained by $C_{c}$ through the clamping of $v_{d s}$ in Off-modes 1 and 2 leads to a worst case analysis without depreciating (17)-(19).

Off-mode $4\left[\boldsymbol{t}_{\mathbf{6}} \sim \boldsymbol{T}\right]$ : This mode is identical to Pre-On-mode 1. The equivalent circuit is shown in Fig. 6(d). This mode ends with the turn-on of $M$ at $t=T$. This completes one switching cycle of the proposed converter.

\section{B. Steady-state analysis}

The finite current slopes due to $L_{k}$ in the proposed converter 


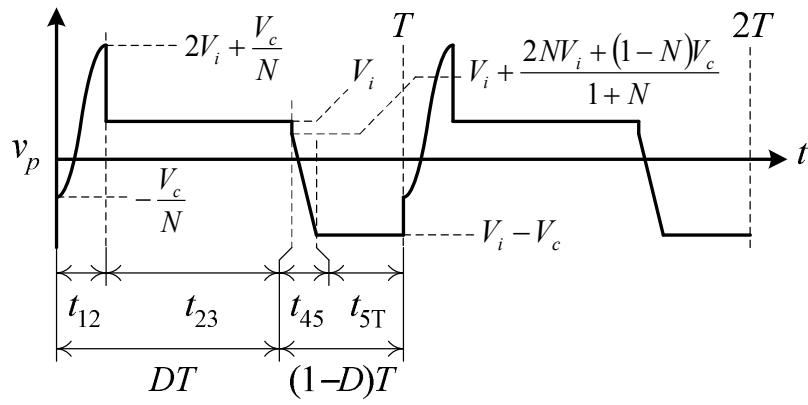

Fig. 7. Waveform of primary winding voltage.

TABLE I

VALUES FOR EACH INTERVAL

\begin{tabular}{c|l|l}
\hline Interval & \multicolumn{1}{|c|}{ Duration } & \multicolumn{1}{|c}{ Average of $\mathbf{v}_{\mathbf{p}}$} \\
\hline $\mathrm{t}_{12}$ & $N \pi \sqrt{L_{k} C_{r}}$ & $V_{i}$ \\
\hline $\mathrm{t}_{23}$ & $D T-t_{12}$ & $V_{i}$ \\
\hline $\mathrm{t}_{45}$ & $2 k C_{r}\left(N V_{i}+V_{c}\right) / I_{M 2}$ & $V_{i}-N\left(V_{c}-V_{i}\right) / k$ \\
\hline $\mathrm{t}_{5 \mathrm{~T}}$ & $(1-D) T-t_{45}$ & $V_{i}-V_{c}$ \\
\hline
\end{tabular}

generate On-mode 1 and Off-mode 1. These modes occupy relatively small portions of one switching cycle. In addition, their contributions to the step-up ratio partially cancel each other out since they reduce both the on-duty and the off-duty. This will be further investigated in part of the next chapter. Therefore, in deriving the steady-state step-up ratio of the proposed converter, these two modes are neglected. Otherwise, the procedure and result become very complicated and do not give very useful insights.

In order to apply the volt-second balance condition on $L_{m}$ for one switching cycle, the voltage waveform across it, denoted by $v_{p}$, is analyzed in Fig. 7, where On-mode 1 and Off-mode 1 are excluded as mentioned above. The duration of each interval and the corresponding average value of $v_{p}$ are shown in Table I. Summing all of the products of the expressions in each row of Table I and equating the result with zero result in:

$$
\frac{2 C_{r} f}{I_{M 2}}\left(N V_{i}+V_{c}\right)^{2}-V_{c}(1-D)+V_{i}=0 .
$$

Since the average voltage across $L_{k}$ in On-mode 2, which can be obtained from (5), is zero, the magnetizing current can be considered to be linearly increasing all the way from $I_{M 1}$ to $I_{M 2}$ during On-modes 2 and 3, that is, $I_{M 2}=I_{M 1}+V_{i} D T / L_{m}$. Then, the output current $I_{o}$ can be obtained by:

i) integrating the secondary current assumed to be linearly decreasing from $I_{M 2} / k$ to $I_{M 1} / k$ during Off-modes 2 4,

ii) integrating the sine term in (19) representing the droop due to the charging current of $C_{c}$ during Off-mode 3,

iii) subtracting ii) from i),

iv) and dividing iii) by one switching period $T$ as:

$$
I_{o}=\frac{1-D}{k}\left(I_{M 2}-\frac{D V_{i}}{2 L_{m} f}\right)-\frac{2 C_{r} f}{k}\left(N V_{i}+V_{c}\right) .
$$

Solving (21) for $I_{M 2}$ and substituting the result into (20) gives:

$$
\frac{V_{c}}{V_{i}}=\frac{1+N \alpha}{1-D-\alpha}, \alpha=\frac{2 C_{r} f\left(\frac{1}{1-D}+N\right)}{\frac{k}{1-D} \frac{I_{o}}{V_{i}}+\frac{D}{2 L_{m} f}} .
$$

Eliminating $V_{c}$ in (22) by using (1) yields the step-up ratio of the proposed converter as:

$$
\frac{V_{o}}{V_{i}}=\frac{1+N D+N \alpha(1+k)}{1-D-\alpha},
$$

where $\alpha$ is as given in (22).

It can be seen from (23) that the parameter $\alpha$ plays a role in increasing the effective duty ratio of the proposed converter. This makes the proposed converter show a higher step-up ratio than the original TIB converter. It can be also seen that a smaller value of $C_{r}$ or a larger value of $I_{o} / V_{i}$ results in a smaller value of $\alpha$. In an extreme case where $\alpha=0$, (23) becomes the step-up ratio of the original TIB converter.

\section{DESIGN CONSIDERATIONS}

Various conditions validating the analysis in the previous chapter are presented in this chapter. Some characteristics of the proposed converter are also discussed in detail. To achieve a seamless flow of the analysis, the validity of (23) will be assumed for the time being. However, in a later part of this chapter, it will be verified by simulation results.

\section{A. No-discharge of $C_{r}$ in On-mode}

In order to make $C_{r}$ not discharge through an extra resonance mode after being charged to its peak voltage in On-mode 2, the sign of (11) is required to be negative as explained in On-mode 2. This condition can be simplified in terms of $V_{o} / V_{i}$ as:

$$
\frac{V_{o}}{V_{i}}>\frac{N+3}{N-1} N
$$

This defines the lower boundary of the step-up ratio. The right-hand side of (24) has a minimum value of 9 at $N=3$ and it becomes larger as $N$ increases or decreases.

\section{B. Continuity of $i_{s}$}

Due to the resonant charging current of $C_{c}$ in Off-mode 3, a current droop appears in the secondary current $i_{s}$ as shown in the $t=t_{5} \sim t_{6}$ interval of Fig. 3. This droop is represented by the sine term in (19). If this droop is too large to maintain the 
secondary current continuous, the proposed converter enters a mode similar to the discontinuous current mode, and the output voltage becomes significantly higher than that predicted by (23). However, this is beyond the scope of this paper, and it should be avoided by the following condition:

$$
k\left(V_{i}+\frac{V_{c}}{N}\right)<I_{M 2} \frac{\sqrt{L_{k} C_{c}}}{C_{r}} .
$$

Eliminating $I_{M 2}$ in (25) using (20) gives a relationship which makes it more convenient to determine the continuity of $i_{s}$ as:

$$
\frac{V_{o}}{V_{i}}<\frac{1+N D+N \beta(1+k)}{1-D-\beta}, \beta=\frac{2 N}{k} f \sqrt{L_{k} C_{c}}
$$

This defines the upper boundary condition for the step-up ratio.

\section{Soft Reset of the Snubber}

If the duty ratio of the proposed converter is too large, the switch can turn on prematurely before $L_{k}$ and $C_{c}$ complete their half-cycle of resonance to make the resonant current in $D_{c}$ zero. In this case, the peak resonant current automatically increases to maintain the charge balance in $C_{c}$ as the switching cycle continues. This does not significantly change the average behavior of the proposed converter over one switching cycle. However, this hard reset of the proposed snubber needs to be avoided since the clamp diode $D_{c}$ in this case fails in achieving soft turn-off which results in an increase in associated side effects such as switching loss and electric noise.

To make sure that the proposed snubber can achieve a soft reset in Off-mode 3, the sum of the time required for $C_{r}$ to completely discharge in Off-mode 2 and the time required for $L_{k}$ and $C_{c}$ to complete a half-cycle of resonance in Off-mode 3 should be shorter than $(1-D) T$. This condition can be obtained from (16) and $\omega_{2}$ in Off-mode 3 as:

$$
\frac{2 k C_{r}}{I_{M 2}}\left(N V_{i}+V_{c}\right)+\frac{N}{k} \pi \sqrt{L_{k} C_{c}}<(1-D) T .
$$

By eliminating $I_{M 2}$ in (27) using (20), another upper boundary condition for the step-up ratio of the proposed converter to ensure the soft reset of the snubber can be obtained as:

$$
\frac{V_{o}}{V_{i}}<\frac{1-D+\frac{k^{2}}{N}-\frac{N(1+k) \pi}{k} f \sqrt{L_{k} C_{c}}}{1-D+\frac{\pi}{k} f \sqrt{L_{k} C_{c}}} .
$$

\section{Characteristic Analysis}

In order to make the analysis of the proposed converter more convenient and illustrative, the practical parameters are assumed to be $V_{i}=12 \mathrm{~V}, f=100 \mathrm{kHz}, N=3, L_{m}=80 \mu \mathrm{H}, C_{c}=330 \mathrm{nF}$, and $C_{r}=4.7 \mathrm{nF}$. Fig. 8 shows the step-up ratio of the proposed

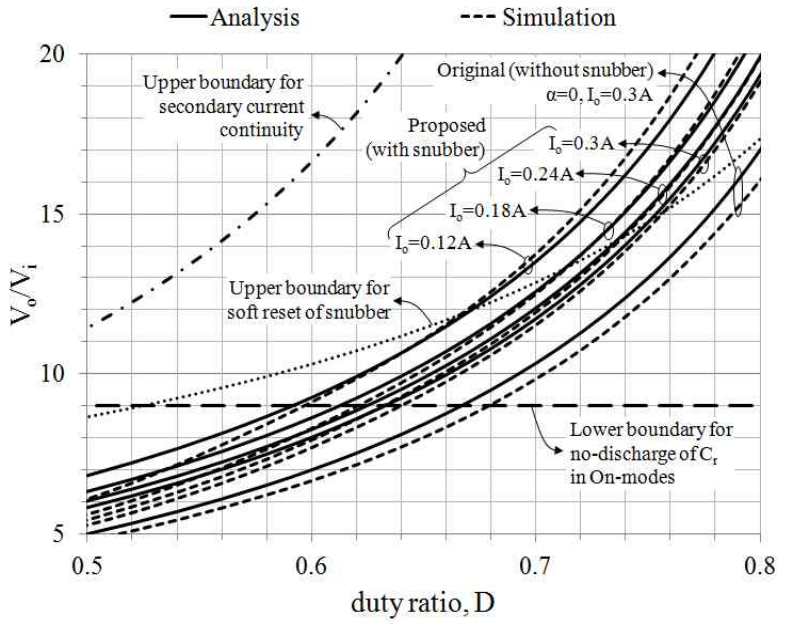

Fig. 8. Step-up ratios and boundary conditions. (a) proposed converter

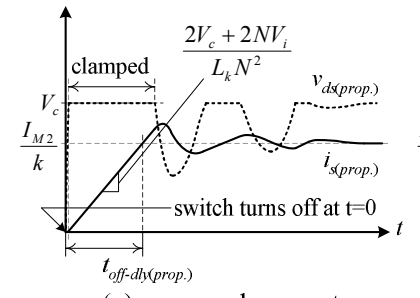

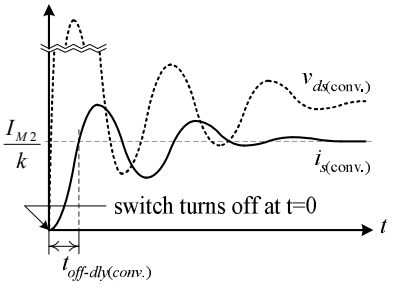

(b) original TIB converter
Fig. 9. Turn-off transient waveforms of $i_{s}$ and $v_{d s}$.

converter in comparison with that of the original TIB converter. The solid lines represent the analytic step-up ratios obtained from (23) for different values of $I_{o}$. The short-dashed lines represent the simulated step-up ratios obtained from SPICE simulations for the same values of $I_{o}$, where $L_{k}=2 \mu \mathrm{H}$ is assumed. The dash-dot and dotted lines represent the upper boundaries given by (26) and (28) for the secondary current continuity and soft reset of the snubber, respectively. The long-dashed line is the lower boundary given by (24) with no discharge of $C_{r}$ during the on-mode. Fig. 8 can be explained from the following standpoints.

1) Effect of $L_{k}$ : Fig. 8 shows that there is a large difference between the analytic and simulated step-up ratios of the conventional TIB converter. This can be explained by using Fig. 9, where the solid and dotted lines represent the secondary current $i_{s}$ and the drain voltage $v_{d s}$ of each converter from the moment the switch turns off. The turn-off delay, denoted by $t_{\text {off-dly, }}$, is defined for each converter by the time required for $i_{s}$ to rise from zero to the steady-state value of $I_{M 2} / k$.

As already explained in Off-mode 1 , the drain voltage $v_{d s}$ of the proposed converter is assumed to be clamped at $V_{c}$ as soon as the switch turns off. The voltage across $L_{k}$ is also clamped since all of the voltages at the tapped-inductor terminals are clamped as shown in Fig. 6(a). Therefore, all of the winding currents vary linearly with slopes determined by the value of $L_{k}$ and the voltage across it. The slope of $i_{s}$ is also shown in Fig. 


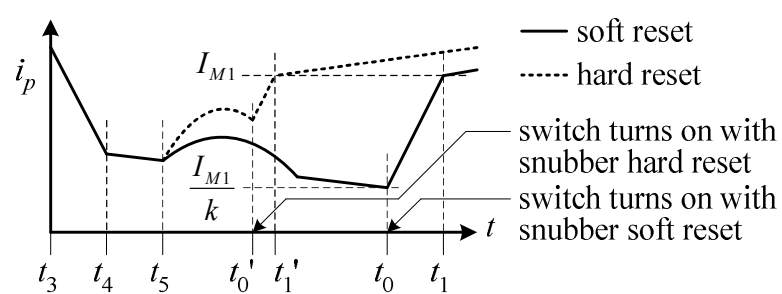

Fig. 10. Soft reset and hard reset of proposed snubber.

9(a). Assuming the voltage across $C_{r}$ is kept constant during the turn-off transient caused by $L_{k}$, the turn-off delay of the proposed converter, denoted by $t_{\text {off-dly (prop.) }}$, can be obtained as:

$$
t_{o f f-d l y(\text { prop. })}=\frac{L_{k} I_{M 2}}{2 k} \frac{N^{2}}{N V_{i}+V_{c}} .
$$

On the other hand, in the original TIB converter, $L_{k}$ and $C_{d s}$ freely resonate after the switch turns off, as shown in Fig. 9(b), since the drain voltage $v_{d s}$ is not limited. The time required for the current $i_{s}$ to reach the steady-state value of $I_{M 2} / k$, denoted by $t_{\text {off-dly(conv.) }}$ is equal to $0.5 \pi\left(L_{k} C_{d s}\right)^{1 / 2}$. This corresponds to a quarter-cycle of resonance. This is much shorter than $t_{\text {off-dly(prop.) }}$ given in (29).

Meanwhile, the turn-on delay, defined by the time required for $i_{s}$ to reach zero after the switch turns on, can be obtained for the proposed converter from (3) as:

$$
t_{o n-d l y}=\frac{L_{k} I_{M 1}}{k} \frac{N^{2}}{N V_{i}+V_{o}} .
$$

Since no snubber elements are associated with (30), the turn-on delay of the original TIB converter can be expressed by (30) and it can be considered equal to that of the proposed converter neglecting a small difference in $I_{M 1}$.

The turn-off delay reduces the off-duty of the converter and adds a small positive volt-second on the primary winding, resulting in a slight increase in the step-up ratio. On the other hand, the turn-on delay reduces the on-duty of the converter and adds a small negative volt-second on the primary winding, resulting in a slight decrease in the step-up ratio. In the proposed converter, the effects of the turn-on delay and the turn-off delay partially cancel each other out since the turn-off delay is relatively large. However, in the original TIB converter, this barely happens since the turn-off delay is very small. This makes the step-up ratio of the proposed converter less affected by $L_{k}$. Therefore, the original TIB converter shows a large difference between the analytic step-up ratio, where $L_{k}$ is neglected, and its simulated counterpart, where $L_{k}$ is taken into account.

2) No discharge of $C_{r}$ in the on-mode: Assuming $N=3$, (24) gives a minimum step-up ratio of 9 , which is shown by the long-dashed line in Fig. 8. Outside this boundary, which corresponds to a step-up ratio lower than 9 , an extra resonance takes place to partially discharge $C_{r}$, resulting in a reduced voltage across $C_{r}$ at the end of the on-modes. This also results in a reduction in the time required for the complete discharge of $C_{r}$ given by (16) in Off-mode 2. As can be seen from Fig. 7, a decrease in $t_{45}$ adds a small negative volt-second on the primary winding, resulting in a slight decrease in the step-up ratio. This is not considered when deriving the analytic step-up ratio of (23). Therefore, a simulated step-up ratio outside this boundary decreases faster with a decreasing $D$ than its analytic counterpart, as shown in Fig. 8.

3) Soft reset of the snubber: Fig. 10 compares the soft reset and hard reset of the proposed snubber. In the soft reset case, the primary current $i_{p}$ starts increasing from $I_{M 1} / k$ at $t=t_{0}$ and reaches $I_{M 1}$ at $t=t_{1}$. In the hard reset case, the switch prematurely turns on at $t=t_{0}{ }^{\prime}$ before $L_{k}$ and $C_{c}$ complete their half-cycle of resonance. Therefore, the primary current starts increasing from a value higher than $I_{M 1} / k$ and reaches $I_{M 1}$ at $t=t_{1}{ }^{\prime}$. Since the slope of $i_{p}$ after the switch turn-on is the same in both cases, the time required for $i_{p}$ to reach $I_{M 1}$ from the switch turn-on is shorter in case of the hard reset. This in effect reduces the turn-on delay defined by On-mode 1 and adds a small positive volt-second on the primary winding, resulting in a slight increase in the step-up ratio. This is shown in Fig. 8, where the boundary condition given by (28) for the soft reset of the snubber is shown by a dotted line. Outside this boundary, the proposed converter performs the hard reset. Therefore, the simulated step-up ratio increases slightly faster with an increasing $D$ than its analytic counterpart in which the snubber hard reset is not considered.

4) Continuity of $i_{s:}$ By comparing the step-up ratio given by (23) and the boundary condition for the continuity of $i_{s}$ given by (26), it can be easily seen that they have very similar forms, and that their traces would also have very similar shapes if they came close to each other. Due to this similarity between (23) and (26), there is no meaningful intersection between them, and the range of the step-up ratio retaining the continuity of $i_{s}$ cannot be well defined. Therefore, this paper suggests designing the proposed converter so that the boundary given by (26) is placed far above the operating condition as exemplified in Fig. 8.

5) Trends of boundary conditions: The analytic step-up ratios of the proposed converter for different values of $N$ are illustrated in Fig. 11 together with all of the boundary conditions presented in this paper. Since the proposed converter is for use in high-step applications, the smallest value of $N$ is chosen to be 3 . The output current is assumed to be $0.24 \mathrm{~A}$, and all of the other parameters are the same to those used in Fig. 8.

As the turns ratio $N$ increases, the minimum duty ratio for no discharge of $C_{r}$ in the on-modes shows a decreasing tendency 


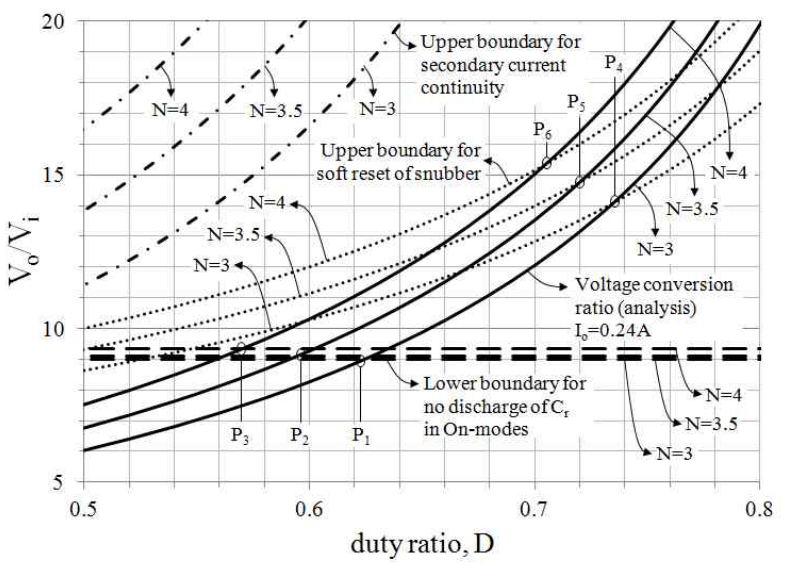

Fig. 11. Trends of boundaries according to $N\left(I_{o}=0.24 \mathrm{~A}\right)$.

TABLE II

KEY PARAMETERS

\begin{tabular}{c|l|l}
\hline Item & \multicolumn{1}{|c|}{ Description } & \multicolumn{2}{c}{ Value } \\
\hline$f$ & Switching frequency & $100 \mathrm{kHz}$ \\
\hline$N$ & Tapped-inductor turns ratio & 3 \\
\hline$L_{m}$ & Primary magnetizing inductance & $80 \mu \mathrm{H}$ \\
\hline$L_{k}$ & Primary leakage inductance & $2.4 \mu \mathrm{H}$ \\
\hline$M$ & Main switch (AOD442) & $60 \mathrm{~V}, 20 \mathrm{~m} \Omega\left(C_{o s s}=155 \mathrm{pF}\right)$ \\
\hline$D_{1}$ & Main diode (ES1G) & $400 \mathrm{~V}, 1 \mathrm{~A}$ \\
\hline$D_{c}$ & Clamp diode (MBRS1100) & $100 \mathrm{~V}, 1 \mathrm{~A}$ \\
\hline$D_{a}, D_{b}$ & Path diodes (MBRS1100) & $100 \mathrm{~V}, 1 \mathrm{~A}$ \\
\hline$C_{c}$ & Clamp capacitor & $330 \mathrm{nF}$ \\
\hline$C_{r}$ & Resonant capacitor & $4.7 \mathrm{nF}$ \\
\hline
\end{tabular}

as denoted by $P_{1}, P_{2}$, and $P_{3}$, and so does the maximum duty ratio for the soft reset of the proposed snubber as denoted by $P_{4}$, $P_{5}$, and $P_{6}$. The boundary for the continuity of $i_{s}$, which is already high enough, becomes even higher as $N$ increases.

\section{EXPERIMENTAL RESULTS}

A prototype of the proposed converter has been built for a $120 \mathrm{~V} / 240 \mathrm{~mA}$ LED lighting module. The input voltage ranges from $9 \mathrm{~V}$ to $12 \mathrm{~V}$ depending on the off-grid power system loaded by a $12 \mathrm{~V}$ battery, and the required step-up ratio ranges from 10 to 13.3. In light of Fig. 11, the tapped-inductor turns ratio is designed as $N=3$, and the operating duty ratio ranges from 0.66 to 0.72 . The switching frequency is chosen to be $100 \mathrm{kHz}$. The tapped-inductor is designed to have a magnetizing inductance of $80 \mu \mathrm{H}$ and it is made with highly productive separate windings to have good insulation and low capacitance between windings. The leakage inductance is measured to be $2.4 \mu \mathrm{H}$. Since the switch voltage is minimized in the proposed converter, a 60V-rated AOD442 with $R_{D S(O N)}=20 \mathrm{~m} \Omega$ is chosen as the switch. A $400 \mathrm{~V} / 1 \mathrm{~A}-$ rated ES1G

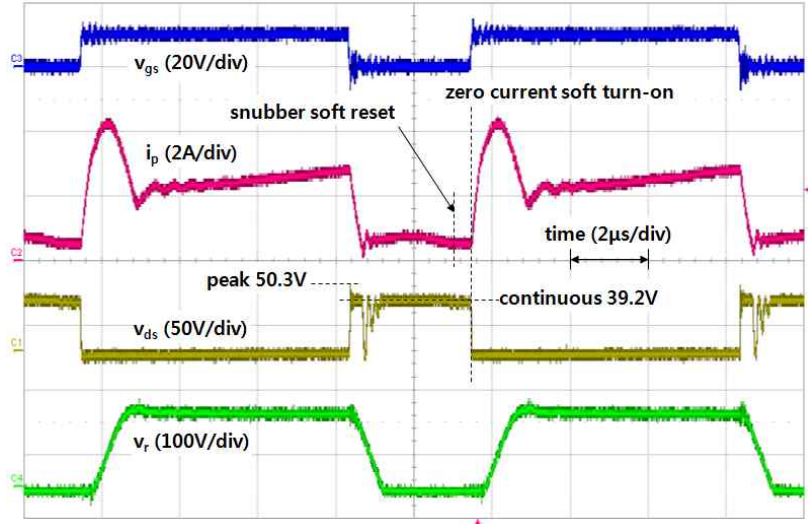

Fig. 12. Key experimental waveforms.

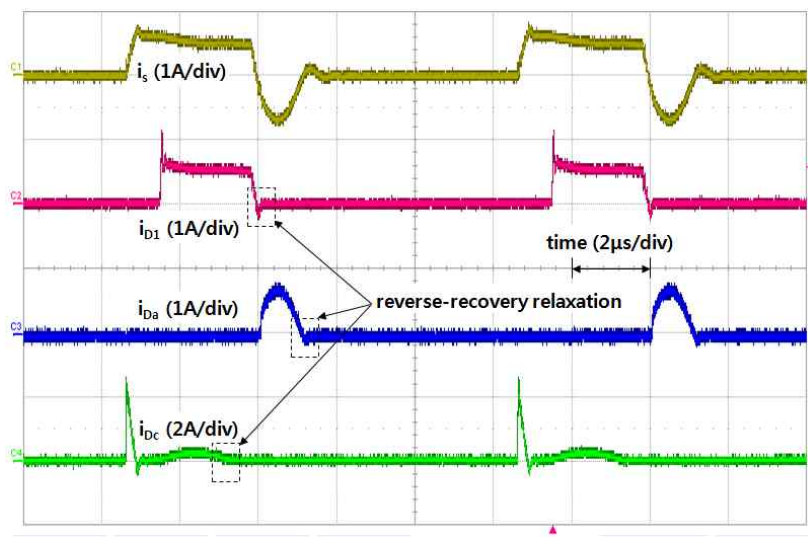

Fig. 13. Additional experimental waveforms.

is chosen as the boost diode, and 100V/1A-rated MBRS1100s are chosen as the clamp and path diodes. The snubber capacitors are designed as $C_{c}=330 \mathrm{nF}$ and $C_{r}=4.7 \mathrm{nF}$. The key parameters are summarized in Table II.

The operating waveforms at the rated input voltage of $12 \mathrm{~V}$ are shown in Figs. 12 and 13. The operating duty ratio is 0.68 , and the measured efficiency is $97 \%$. The continuous switch drain voltage is measured to be $39.2 \mathrm{~V}$ as expected by (1), and the peak switch drain voltage, which is caused by parasitic inductances between the components, is measured to be $50.3 \mathrm{~V}$. All of the waveforms match well with the theoretical waveforms shown in Fig. 3. In addition, the operation and features of the proposed converter such as the minimum switch voltage stress, soft-switching of the switching devices, relaxation of the diode reverse-recoveries, and the soft reset of the snubber are all verified.

For a comparison with the original TIB converter without a snubber, the switch of the proposed converter is replaced by a $200 \mathrm{~V}$-rated AOD2210 with $R_{D S(O N)}=105 \mathrm{~m} \Omega$. The efficiency is $94.5 \%$, which is lower than the previous result of $97 \%$. This is due to increased conduction loss since the AOD2210 has a higher $R_{D S(O N)}$ than the AOD442. The proposed converter is then converted into the original TIB converter by removing all 
TABLE III

MEASURED EFFICIENCY

\begin{tabular}{c|c|c|c|c}
\hline \multirow{2}{*}{ Topology } & Switch & \multirow{2}{*}{ Efficiency } & \multicolumn{2}{|c}{ Key loss factor } \\
\cline { 5 - 5 } & & $\begin{array}{c}\text { Switch } \\
\text { turn-on loss }\end{array}$ & $\begin{array}{c}\text { Switch } \\
\text { conduction loss }\end{array}$ \\
\hline Proposed & AOD442 & $97 \%$ & $\sim 0 \mathrm{~W}$ & $\begin{array}{c}0.16 \mathrm{~W} \\
(60 \mathrm{~V} / 20 \mathrm{~m} \Omega)\end{array}$ \\
\hline Proposed & $\mathrm{AOD} 2210$ & $94.5 \%$ & $\sim 0 \mathrm{~W}$ & $\begin{array}{c}0.81 \mathrm{~W} \\
(200 \mathrm{~V} / 105 \mathrm{~m} \Omega)\end{array}$ \\
\hline Original & $\mathrm{AOD} 2210$ & $91.5 \%$ & $\begin{array}{c}0.68 \mathrm{~W} \\
(2.4 \%)\end{array}$ & $\begin{array}{c}0.83 \mathrm{~W} \\
(2.9 \%)\end{array}$ \\
\hline
\end{tabular}

of the snubber elements and adjusting the duty cycle to be 0.72 to maintain the output voltage. An extra $470 \mathrm{pF}$ capacitor is added between the switch drain and source to keep the voltage spike from becoming too high by exceeding $600 \mathrm{~V}$. The efficiency of this original TIB converter is measured to be $91.5 \%$, which is lower than the previous result of $94.5 \%$. This is due to the increased switching loss. The results are summarized in Table III. The key loss factors affecting the results are also shown in the table.

\section{CONCLUSIONS}

For emerging high step-up applications in off-grid areas, a passive lossless snubber for the continuous current mode TIB converter is proposed. In the proposed converter, the switch voltage is minimized so that a more inexpensive and efficient device can be selected. Soft-switching is also provided for the switching devices, resulting in a further improvement in the efficiency. Since the leakage inductance is fully utilized, there is no need to minimize it, which allows for a cost-effective and highly productive design of the tapped-inductor. In addition, the proposed converter has a high step-up ratio and provides relaxation of the diode reverse-recovery.

In this paper, the operation principle and characteristics of the proposed converter are analyzed in detail. In addition, various boundary conditions that need to be considered in the design are presented. Some experimental results are also presented to confirm the operation and features of the proposed converter.

\section{REFERENCES}

[1] S. Misak and L. Prokop, "Off-grid power systems," in Proc. EEEIC, pp. 14-17, 2010.

[2] D. Frame, K. Tembo, M. J. Dolan, S. M. Strachan, G. W. Ault, "A community based approach for sustainable off-grid PV systems in developing countries," in Proc. IEEE Power and Energy Society General Meeting, pp. 1-7, 2011.
[3] A. A. Freitas, S. Daher, F. Antunes, S. Ximenes, F. Viana, 1, E. Sá Jr, F. S. Silva, and E. A. Soares, "Off-grid PV system to supply a rural school on DC network," in Proc. ICREPQ, paper number 535, 2010.

[4] Clean Energy Ministerial -Global LEAP awardshttp://www.globalleapawards.org, 2013.

[5] N. Vazquez, L. Estrada, C. Hernandez, and E. Rodriguez, "The tapped-inductor boost converter," in Proc. ISIE, pp. 538-543, 2007.

[6] D.-H. Jang, J.-I. Kang, and S.-K. Han, "High efficiency lossless snubber for photovoltaic maximum power point tracker," Trans. KIPE, Vol. 18. No. 5, pp. 485-491, Oct. 2013.

[7] M. Nakamura, K. Ogura, and M. Nakaoka, "Softswitching PWM boost chopper-fed dc-dc power converter with load side auxiliary passive resonant snubber," Journal of Power Electronics, Vol. 4, No. 3, pp. 161-168, Jul. 2004

[8] T. Ahmed, S. Nagai, E. Hiraki, and M. Nakaoka, "A new three winding coupled inductor-assited high frequency boost chopper type dc-dc power converter with a high voltage conversion ratio," Journal of Power Electronics, Vol. 5, No. 2, pp. 99-103, Apr. 2005.

[9] R.-J. Wai and R.-Y. Duan, "High step-up converter with coupled-inductor," IEEE Trans. Power Electron., Vol. 20, No. 5, pp. 1025-1035, Sep. 2005.

[10] W. Dong, Q. Zhao, J. Liu, and F. C. Lee, "A boost converter with lossless snubber under minimum voltage stress," in Proc. APEC, pp. 509-515, 2002.

[11] C.-J. Tseng and C.-L. Chen, "A passive lossless snubber cell for nonisolated PWM DC/DC converters," IEEE Trans. Ind. Electron., Vol. 45, No. 4, pp. 593-601, Aug. 1998.

[12] Q. Zhao and F. C. Lee, "High-efficiency, high step-up DC-DC converters," IEEE Trans. Power Electron., Vol. 18, No. 1, pp. 65-73, Jan. 2003.

[13] C.-J. Tseng and C.-L. Chen, "Passive lossless snubbers for DC/DC converters," IEE Proc.-Circuits, Devices and Systems, Vol. 145, No. 6, pp. 396-401, Dec. 1998.

[14] J.-M. Kang, S.-H. Lee, S.-S. Hong, and S.-K. Han, "Voltage clamped tapped-inductor boost converter with high voltage conversion ratio," Trans. KIPE, Vol. 17, No. 1, pp. 34-40, Feb. 2012.

[15] R. T. H. Li and H. S.-H. Chung, "A passive lossless snubber cell with minimum stress and wide soft-switching range," IEEE Trans. Power Electron., Vol. 25, No. 7, pp. 1725-1783, Jul. 2010.

[16] K. M. Smith Jr. and K. M. Smedley, "Engineering design of lossless passive soft switching methods for PWM converters. I. with minimum voltage stress circuit cells," IEEE Trans. Power Electron., Vol. 16, No. 3, pp. 336-344, May 2001.

[17] H. Levy, I. Zafrany, G. Ivensky, and S. Ben-Yaakov, "Analysis and evaluation of a lossless turn-on snubber," in Proc. APEC, pp. 757-763, 1997

[18] D.-H. Kim, J.-H. Jang, J.-H. Park, and J.-W. Kim, "Single-ended high-efficiency step-up converter using the isolated switched-capacitor cell," Journal of Power Electronics, Vol. 13, No. 5, pp. 766-778, Sep. 2013.

[19] J.-i. Kang, S.-K. Han, and J. Han, "Lossless snubber for tapped-inductor boost converter for high step-up application," in Proc. ICIT, pp. 253-260, 2014. 


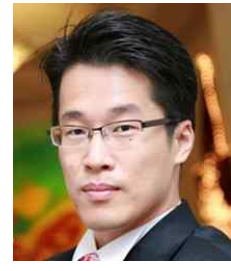

Jeong-il Kang received his B.S., M.S., and $\mathrm{Ph} . \mathrm{D}$. degrees in Electrical Engineering from the Korea Advanced Institute of Science and Technology (KAIST), Daejeon, Korea, in 1995, 1997, and 2002, respectively. In 2002, he joined Samsung Electronics Co., Ltd., Suwon, Korea, and is currently working on the design and control of semiconductor light source drivers for various display and lighting devices as a Principal Engineer in the Visual Display Research \& Development Office. His current research interests include the development, modeling, and control of power converter topologies. He is a Member of the Korean Institute of Power Electronics (KIPE).

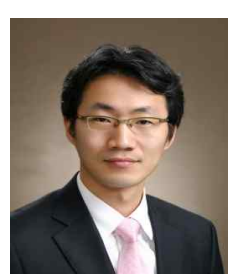

Sang-Kyoo Han received his M.S. and Ph.D. degrees in Electrical Engineering and Computer Science from the Korea Advanced Institute of Science and Technology (KAIST), Daejeon, Korea, in 2001 and 2005 , respectively. For the next six months, he was a Post-Doctoral Fellow at KAIST where he developed digital display power circuits and preformed several research activities. Since 2005, he has been with the Department of Electrical Engineering, Kookmin University, Seoul, Korea, as an Associate Professor. He has also worked for the Samsung Power Electronics Center (SPEC) and the Samsung Network Power Center (SNPC) as a Research Fellow. His primary areas of research interests include power converter topologies, LED drivers, renewable energy systems, and battery chargers for electric vehicles. Dr. Han is a Member of the Korean Institute of Power Electronics (KIPE).

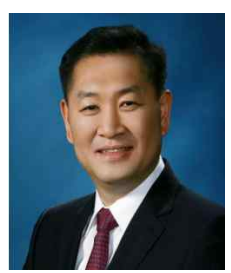

Jonghee Han is an Executive Vice President of the Visual Display Business for Samsung Electronics. In this role, he leads the Visual Display Research \& Development Office. Since joining Samsung in 1988, Mr. Han has played a significant role in the success of Samsung's Visual Display business, helping it solidify its top position within the consumer electronics industry. One of his many successes includes the launch of the award-winning 'Bordeaux' TV series, which became one of the world's best-selling LCD TVs in 2006. During his tenure, Mr. Han has accomplished a number of "firsts" in the TV industry, serving at the forefront of some of the most noteworthy TV advancements. For example, he was an integral force in the development of the world's first 3D LED TV in 2010 and first Smart TV in 2011, among numerous other developments. More recently, he played a critical role in the creation of both the world's first Curved OLED TV and Curved UHD TV. In particular, his research specialties include image processing, audio signal processing, analog/digital mixed systems, display devices, and power electronics for both innovative displays and audio products. Along with contributing his vast knowledge to Samsung's Visual Display Business, he is also a Member of the Korean Institute of Power Electronics (KIPE). Mr. Han holds a bachelor's degree in electrical engineering from Inha University, Incheon, Korea. 